\section{Briginal Articlet.}

\section{CHRONIC TEA POISONING. ${ }^{1}$}

BY WILLIAM N. BULlaRd, M.D., BostoN

Gentlemen, I desire to-night, so far as lies in my power, to draw your attention to an affection which from its great frequency, the peculiar discomfort of its symptoms and its relative curability, is worthy of the careful consideration of the profession. At first sight it may seem surprising that so little has been either written or spoken in regard to this subject, but I believe this to be principally due to two causes, firstly, the apparent ease with which any symptoms thus produced could be checked, by withdrawal of the existing agent, and secondly, to the indefiniteness and obscurity of these symptoms and the difficulty of proving their origin. During my study of this subject $I$ have become more and more convinced that the latter difficulty is our greatest obstacle to a thorough knowledge of this affection, and that it is only to be overcome by the most careful investigation of each individual case and by the comparison of statistics drawn from a considerable number of such cases.

In the literature of this subject, which is curiously scanty and much of which has been written by the laity or from a non-professional point of view, I have been able to find only general statements, but few definite proved facts, and no statistics.

In this paper we desire to confine our consideration to the cases of ordinary sub-acute and chronic tea-poisoning, putting aside all acute cases, where large quantities of tea have been imbibed at a single sitting, and all those of tea-tasters or persons by profession exceptionally exposed to its toxic influence. In both these classes the symptoms are plain and well-known, and Morton's paper on tea-tasters leaves little more to be done in that direction except by those having special opportunities. The cases which I wish to consider are of the sort which present themselves daily to the general practitioner with symptoms sometimes obscure, sometimes more or less pronounced.

The first fact which strikes the observer is the very gradual and insidious growth of the symptoms, which at first are frequently so slight and so indefinite as to be scarcely noticeable and only produce serious discomfort after a considerable period. This is one cause why their origin is not recognized by the patients themselves, and another reason for this may be found in the fact that the majority of them are of so general a character that they can readily be referred to some other source. Hence arises the difficulty in diagnosis, which so frequently occurs, and there are many cases in which it remains impossible to determine how far certain symptoms are the result of tea and how far they are due to other causes. As the cases become more severe the symptoms become more distinct and the diagnosis easier.

I will first describe a few cases of typical tea-poisoning, and then proceed to the analysis of the symptoms.

Case I. Boston Dispensary. Thomas G., about fifty-five years old, a horse-car washer. Complains of attacks of " rolling in the stomach" followed by flatus and tenesmus, and has attacks of cold chills and weakness with a tendency to faint. He has staggered sev-

1 Read before the Section of Clinical Medicine, Pathology and $\mathrm{Hy}$ giene of the Suffolk District Medical Society, March 10, 1886. eral times and come near fainting while at work, but has never fainted away. Has lost flesh lately. No palpitation: no cough. Drinks from one to three pints of tea per diem. He is emaciated and has a cachectic appearance. The heart and lungs are normal, so far as can be detected, except that the second sound is accentuated in the region of the pulmonary artery. Urine is normal in appearance, contains no albumen. Patient has lumbago and probably hæmorrhoids.

Case II. Carney Hospital. Woman, forty years old. Diagnosis, "tea-dyspepsia." Patient is markedly nervous. She feels excited " as if afraid of somebody," especially when in a crowd. She used to have vertigo, but has never fallen. For two years she has been subject to distress in the epigastrium, palpitation, constipation, a dull heavy headache and dizziness, no cough. She drinks tea three times a day and also between meals.

Case III. Woman, twenty-three years old. Diagnosis, "tea-poisoning; anæmia." This patient applied at the Carney Hospital Out Patient Department on March 19th, 1885, on account of bilateral headaches, frontal and occipital, accompanied by nausea and vomiting, which had occurred once a week for the preceding six months. She had been subject to these previously, but they were less severe and for about two years had been entirely absent. Her appetite was poor and she had a feeling of "weakness" in the epigastrium, but there was no distress after food, bowels regular. Had palpitation. Had never had rheumatism. Drank tea three times a day and also between meals. Under proper treatment she reported herself well on the $2 \mathrm{~d}$ of April.

Case IV. Girl, thirteen years old. Complains of pain in both sides, especially the left, nausea, but no vomiting, headache frontal and bilateral, palpitation and dyspnoea. Bowels constipated. The heart and lungs were normal, as far as could be detected. There was some tenderness over the epigastrium. Drank one to one and a half cups of tea three times a day. This is a typical case of light tea-poisoning.

Ease V. Woman, sixty years old. Dull frontal headache followed by "numbness" all over, which seems to be a sensation of weakness rather than a paræsthesia. Attacks of dizziness, when she fears that she will fall. Appetite poor. No vomiting, but nausea with the headache. Bowels regular. Drinks pure black tea strong three times a day and occasionally between meals. Distinct improvement in a week under treatment.

Morton in his article above referred to, gives the following symptoms as the result of the "continued and immoderate use of tea: "headache, ringing in the ears, tremulousness, " nervousness," exhaustion of mind and body with disinclination to mental and physical exertion, increased and irregular action of the heart, and dyspepsia. Starting from this as a basis for investigation, it will be interesting to see how nearly his symptoms correspond to those in the class of cases under consideration. Morton's deductions are drawn from five cases, all tea-tasters.

Our statistics are based on one hundred and sixtythree cases of tea-drinkers, twenty-seven from the Boston Dispensary, one hundred and thirty-six from the Out Patient Department of the Carney Hospital. In addition we have examined for the purpose of differentiation one hundred and fifty-eight cases in which 
symptoms resembling those of tea-poisoning occurred, but in which we were not inclined to attribute the symptoms to the abuse of tea. All the cases from the Boston Dispensary were collected from one of the Women's Rooms, and were consequently adult females. Putting these aside we find that out of the one hundred and thirty-six cases at the Out Patient Department of the Carney Hospital, where men, women and children are all received, by far the larger proportion are in women. The numbers are as follows: men, nine; children under fifteen, twelve; (boys three, girls nine); women, one hundred and fifteen.

Let us now consider the frequency of the different symptoms in our patients and determine, as nearly as possible, what combination of these may be accepted as typical of chronic tea-poisoning. We will follow the order of symptoms given by Morton. It must be stated, however, that in many cases only the more prominent symptoms were noted, and that the existence of the less marked has probably sometimes been overlooked.

1. Headache. This occurred in forty-nine cases (thirty per cent.). Hemicrania existed in eleven cases (six per cent.); in the others the pain was bilateral and situated generally in the forehead and temples.

2. Ringing in the ears was not frequent. It was noted in but three cases, in two of which it was due to disease of the ear, while in the third case it accompanied a congestive headache supposed to have been due to exposure to a hot sun. It cannot consequently be considered as a symptom of chronic tea-poisoning.

3. Tremulousness. This is not uncommon, and it probably occurred to a slight extent in many cases where its existence was not specified, while in others it was included under the title of nervousness. It does not, however, appear to be as common as might be expected and it is certain that in many cases it does not occur. Tremor is noted in six cases, but in only one of these does it seem fairly attributable to the tea. Hence we conclude that tremor is not an ordinary symptom of chronic tea-poisoning.

4. Nervousness. By this term we understand an undue excitability or irritability of the nervous system, both as shown subjectively by a general sensation of uneasiness and restlessness, and as evidenced objectively by an exaggerated reaction to sudden external impulses, as loud sounds, bright lights, especially when unexpected, and by a diminution in the normal power of control over the emotions, for example, an increased tendency to emotional display, laughing or crying. Nervousness is noted in twenty-seven cases (sixteen per cent.), its absence in one. Slight degrees of nervousness probably exist in nearly all these patients, but we cannot, therefore, conclude that it is the direct result of tea ; on the contrary, in many cases, it is undoubtedly due to the concomitant digestive troubles. It has only been noted where it was a prominent and wellmarked symptom.

5. Exhaustion of Mind and Body, with Disinclination to Mental and Physical Exertion. This symptom undoubtedly exists in many cases, but is of too indefinite a character to be susceptible of accurate determination in the class of cases with which we have to deal. Moreover, its liability to occur from other causes renders it impossible to determine how far it is due to tea in our patients. General weakness is mentioned as a prominent symptom in thirteen cases (eight per cent.). All cases of anæmia are excluded.
6. Increased and Irregular Action of the Heart. I had expected to find this in nearly every case, but, although frequent, it does not appear to be a necessary concomitant of tea-poisoning in this form. Palpitation is recorded in eighty cases (forty-nine per cent.) It is present in almost all severe cases. Irregularity of the heart occurred in six additional cases. In four cases where it would naturally be expected, the absence of palpitation is noted.

7. Dyspepsia. The occurrence of gastric or intestinal symptoms with chronic tea-poisoning is almost universal ; but on the other hand, in many cases these do not assume a very prominent form. Under this heading, we have noted only cases in which there was a distinct sense of oppression or fulness in the epigastriun after eating. In this sense, we find that there were seventy-five cases (forty-six per cent). Pain in the epigastrium occurred in sixteen cases (nine per cent.), six of which were included under dyspepsia.

Vomiting occurred in twenty-eight cases (seventeen per cent.); nausea in thirty-two (twenty per cent). Twenty-two of the cases of vomiting had been noted under dyspepsia; in thirteen cases it was combined with nausea. In one case only was it noted separately, and in this it occurred with a headache, which was sometimes unilateral.

Nausea was, in twenty-three cases, combined with dyspepsia ; in thirteen, with vomiting. In five cases it is reported alone. In three of these it occurs with headaches, in one there is trigeminal neuralgia, and in the last it is probably due to tobacco.

Pain in the left side or in the cardiac region, so frequent a symptom in women, and which, in most cases, is probably due to intestinal conditions, occurred in but thirty-four cases (twenty-one per cent). It can, therefore, scarcely be considered as exceptionally frequent in this class.

Intestinal disturbances of any serious character do not occur in chronic tea-poisoning. Before examining this series of cases, we had supposed that we should find constipation to be a marked and definite accompaniment of habitual and excessive tea-drinking, but as we compared the symptoms in the various cases more closely, we were more and more surprised to find how little foundation existed for this opinion. As a matter of fact, we find that out of our one hundred and sixty-three cases, seventy-seven only (forty-seven per cent.) are recorded as having been subject to constipation; while in forty-six (twenty-eight per cent.), the bowels are recorded as regular, and in three cases there was looseness. Of the thirty-seven cases not recorded, it is but fair to suppose that no marked constipation existed, because this was carefully looked for and noted in every case, while in the more lately recorded cases, its absence was specially noted. In one hundred cases of women taken from the same clinic, but not affected with tea-poisoning, precisely fifty were recorded as suffering from constipation.

The condition of the appetite was found to be quite different in different cases, more frequently somewhat affected. Yet in some of the severest cases it was absolutely unchanged, and occasionally it seemed to be even increased. Poor appetite is recorded in fifty-four cases (thirty-three per cent.); appetite good or fair in twenty (twelve per cent.); excessive in one. Only nineteen of the cases of loss of appetite coincide with those of dyspepsia, leaving forty-seven cases of dyspepsia in which the condition of the appetite was not re- 
corded, and it seems fair to presume that in most of these the appetite was more or less affected. Adding these forty-seven cases to the fifty-four before mentioned, we have a total of one hundred and one cases (sixty-one per cent.). This proportion cannot be much too large.

We now pass to symptoms not mentioned by Morton, which occur more or less frequently in our patients.

Dyspncea occurs in a considerable number of cases, twenty-three (fourteen per cent.), but seems usually to be secondary to anæmia or cardiac weakness. In two of the cases it was evidently due to accompanying pulmonary disease. In nineteen it was accompanied by palpitation.

The general conditions of health varied considerably in the patients examined. Most of them were welldeveloped, and in few cases was there evidence of any serious organic disease. Anæmia was present in twenty-nine cases (eighteen per cent.).

In regard to the condition of the nervous system in this class of cases, we hope to speak more fully on some other occasion. There is no question but that there exists among these patients exaggerated nervous excitability and irritability, and a distinct tendency to functional nervous affections, both of an hysterical and of a neuralgic type. Nervousness, as before mentioned, was noted in about sixteen per cent. Neuralgia existed in twenty-one cases (thirteen per cent.); herpes zoster in one. We find distinct nervous symptoms, not including palpitation or bilateral headaches, which are frequently of dyspeptic origin, in sixty-eight cases (forty-two per cent.).

To sum up these, we conclude from the figures presented, that the most common symptoms in chronic teapoisoning are loss of appetite, dyspepsia, palpitation, headaches, nervousness, and various forms of functional nervous affections of an hysterical or neuralgic character, vomiting and nausea. The constipation, which so frequently occurs, cannot be considered due to the tea.

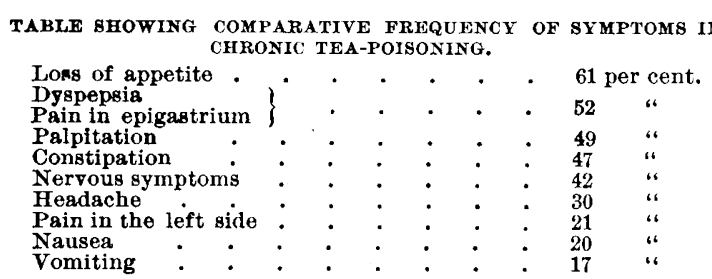

All these symptoms are common in many other diseases, but it is the special combination, which exists here, and above all, the predominance of the nervous group, which enables us to form our diagnosis. It is evident that there exists many cases of this affection in which no diagnosis could be arrived at from the symptoms alone without the history, but I firmly believe that in the majority of the cases, a presumption in favor of this affection is justifiable from the sole evidence of the symptoms.

The first evidence of chronic tea-poisoning is usually given by symptoms referable to the digestive organs. We notice a gradually increasing difficulty in digestion; as shown by a sense of weight and heaviness in the epigastrium, uneasiness after taking food, and general lowering of tone, both mental and physical. If the habit of tea-drinking be continued, a general restlessness and excitability of the nervous system makes its appearance, soon followed by a marked disinclination for food, and by headaches. Constipation is very common. Palpitation, which has previously been slight, now becomes distressing, and is not infrequently accompanied by dyspnoca. Up to this point, the onset of the symptoms has been gradual, and none of them have been severe, so that the patient, if asked, would almost always declare herself in good health. In the more severe cases the previous symptoms become aggravated, the headaches become more or less severe, and more or less persistent, and neuralgias and other symptoms referable to the nervous system, paræsthesia, anæsthesia, and various forms of functional cerebral trouble, show themselves : the latter are due in part, perhaps, to the digestive derangements, which are especially liable to betray themselves by vomiting and nausea. In other cases, the symptoms point rather to the class known as hysteria.

As to the anæmia which exists in many cases, I am inclined to regard it as quite as much cause as result of the tea-poisoning. In many cases it is probably both.

If we now analyze the classes of symptoms in detail shall we find any points - except that of combination - by which we can distinguish them from the same classes of symptoms in other diseases? At present, at least, until we are able to examine more carefully into the smaller details, this must be answered in the negative. Nevertheless there are certain points to which it may be well to cali attention, inasmuch as they may serve as a basis for future more thorough investigation. We will therefore consider these classes in turn. Digestive Symptoms. These should be divided into gastric and intestinal. In making the division we are at once struck with the predominance of the gastric symptoms both in frequency and severity. With the exception of constipation and pain in the left side, which do not occur more frequently in these patients than in others of their class, who are unaffected by tea, intestinal symptoms are rare. Only four cases are found in which they exist so as even to be noted, and in only one of these is it probable that they had any connection with tea.

The ordinary symptoms of gastric disturbance, pain and oppression in the epigastrium, distress after food and in severe cases nausea and vomiting are compara. tively frequent, and in connection with these should be placed certain forms of headache, which are evidently secondary to gastric disturbance. These symptoms are not to be distinguished from those due to ordinary subacute or chronic dyspepsia arising from other causes. There are, however, certain forms of dyspepsia, or perhaps more properly of gastroneurosis, which should at once direct our attention to the probability of tea-poisoning. These occur more markedly in the severe cases and are characterized by the fact that the gastric trouble seems to depend rather on some abnormal conditions of the nerves than on any dyspepsia, properly so-called. Wherever a marked gastralgia exists without evidence of organic disease, wherever we find frequent vomiting apparently unaffected by food and unaccompanied by gastric catarrh ; in other words, wherever we find evidence of a true gastroneurosis, or of increased excitability of the gastric nerves greater than could ordinarily be accounted for by the condition of the stomach, whether dyspepsia be present or not, if the agency of alcohol can be eliminated, we should always suspect tea-poisoning.

In regard to the symptom of pain in the left side or 
in the cardiac region, it is in many cases difficult to determine from the character and position of the pain alone whether it be due to cardiac trouble or to intestinal disturbance. As far as my observation goes I incline to think that the latter cause is the most common, but on this point I have no satisfactory data.

Circulatory Apparatus. The most prominent symptoms relating to the circulatory apparatus are palpitation and irregularity of the heart-beat. These occur frequently and our attention is often first drawn to the cause of the affection by their presence. They usually occur together; indeed, I believe that in all cases of palpitation more or less cardiac irregularity may be detected. They are undoubtedly due in most cases to reflex action on the nerves of the heart, and may fairly be considered as evidence of a functional affection of the nervous system of the heart or a cardiac neurosis.

We have at present no evidence in regard to the condition of the blood-vessels in this affection, and it is reasonable to suppose that no great variation from the normal will be found, inasmuch as the trouble is so chronic and in many cases so slight. Whether or not any increase exists in the excitability of the vaso-motor nerves under these conditions would be an interesting subject for investigation.

Pulmonary Symptoms. The only pulmonary symptom which exists in chronic tea-poisoning is dyspnœa, and this is probably always secondary to cardiac weakness and irregularity. We have thus far no evidence of any affection of the pulmonary branches of the pneumogastric.

Symptoms Referable to the Nervous System. It is the great prominence of functional affections of this class which, in combination with the symptoms already described, gives its peculiar character to the symptom-complex of tea-poisoning. All these symptoms are met with frequently in many and very various diseases, and it is only through the special method of their combination that any distinctiveness is attained. Now it is precisely these functional affections of the nervous system which, when they take a prominent position in connection with the ordinary symptoms of dyspepsia, should lead us to consider the probability of the toxic action of tea. What these affections are I hardly need state, especially as I desire to enter more fully into this subject at some future time, but among the more common forms are paræsthesiæ, neuralgias and functional motor paralyses, usually temporary. It is of special importance to remark that the affections of the nervous system due to chronic tea-poisoning are, so far as my observations show, always functional. Where organic symptoms exist they are not connected with tea.

Having now considered in more or less detail the symptoms produced by tea when taken in excess for a considerable period of time, we are naturally led to the inquiry under what conditions does tea exert this toxic influence and what amount is usually required to cause it. It is evident that the answer to the latter question depends on the character (strength and quality) of the tea used. The class of persons with whom we have to deal, as a rule, use either Oolong or English Breakfast tea, or a mixture of the two. Stronger kinds are sometimes, but rarely, used.

In regard to the amount necessary to produce chronic intoxication, this naturally varies according to the physical condition of the subject and the period during which its use has been maintained. In order to ob- tain an approximate estimation thereof we have tabulated the amounts taken daily by 171 patients, 94 of whom had no symptoms of tea-poisoning, while in 77 these symptoms were present. In estimating the amounts we are perfectly aware that absolute accuracy is not obtainable, if for no other reasons, at any rate because in the first place the teacups used vary in size, and secondly the strength of the infusion cannot be determined.

Our results are as follows : 94 female patients of the same class as the tea-drinkers, but in whom there were no distinctive tea-symptoms, who applied at the Carney Hospital during this period, were questioned in regard to the amount of tea drunk, in order that a standard of comparison might be obtained. Some of these were suffering either from neuralgia or dyspepsia which might have been caused, at least in part, by their addiction to tea, but there was no proof of its influence. Out of these patients,

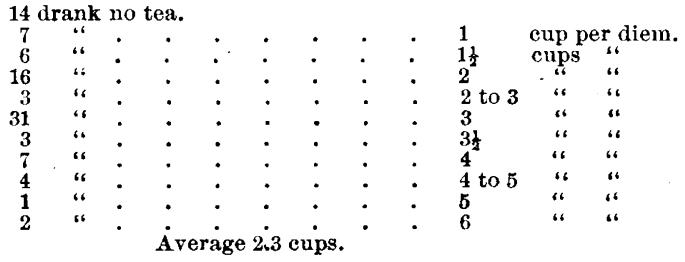

Seventy-four patients with symptoms of tea-poisoning drank tea as follows:-

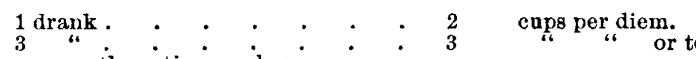

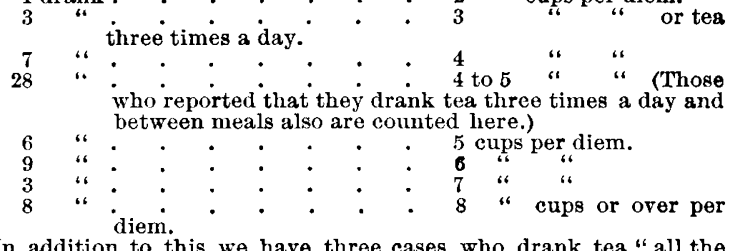

In addition to this we have three cases who drank tea "all the time."

From these figures it appears that those in whom toxic symptoms were present drank, as a rule, much more tea than those in whom these symptoms did not appear, which tends to prove that these symptoms were, as we have previously stated, caused by the tea. ${ }^{2}$ In the second place we find that, as a rule, about five cups a day are required by the average patient of this class to produce chronic intoxication.

The physical condition of the subject, apart from any natural idiosyncrasy, has an important bearing on the action of tea upon the system. Youth, anæmia, or weakness from any cause, deprivation of fresh air and exercise and want of other food all tend to increase its deleterious effects. Thus we find that infants and all persons under the age of puberty are affected with ease. Again, weakness of any kind and anæmia naturally render the system more susceptible to the injurious effects of external agents as the normal power of resistance is wanting, and hence we find that even those adults who have for years been accustomed to take a moderate amount of tea without evil effects, if for any reason they become anæmic, if their strength be exhausted by excessive work or in other ways, immediately begin to show toxic symptoms, although the amount of tea ingested has not increased. This is

2 It seems scarcely necessary for me to state that the diagnosis in all the cases had been made and entered on the books sometime before this table was thought
involuntary self-deception. 
well shown in cases of chlorosis, and is very common among factory-operatives and seamstresses. We desire to lay special stress on this fact, which is one cause of the non-recognition of the toxic agency of tea by the patient.

That deprivation of fresh air and want of sufficient food should cause the same result is only to be expected, not only because in themselves they tend directly to produce weakness and anæmia, but also because the toxic products are not so readily eliminated. It is for the same reason that more tea can be drunk without evil effect by persons when working or taking exercise than could be taken by the same person when idle or at rest.

In conclusion, I would state that $\mathrm{I}$ have arrived at the following results :

(1) That the action of tea is cumulative.

(2) That its action is more pronounced on the young and on those subject to anæmia or in a depressed physical condition, although persons otherwise healthy not infrequently show toxic symptoms.

(3) That among the class of people under consideration, who, as a rule, use medium grades of Oolong and English Breakfast tea the average amount needed to cause toxic symptoms is a little less than five cups per diem.

(4) That chronic tea-poisoning is a frequent affection, and that its most common symptoms are loss of appetite, dyspepsia, palpitation, headache, vomiting and nausea, combined with nervousness and various forms of functional nervous affections, hysterical or neuralgic. These symptoms are frequently accompanied by constipation and pain in the left side or cardiac region.

\section{RECENT PROGRESS IN THE TREATMENT OF THE INSANE.' \\ BY WALTER CHANNING, M.D.}

DRUGS IN THE TREATMENT OF THE INSANE.

Dr. STARK of Illenau has written a paper on the "Present Status of the Paruldehyde Question," 2 characterized by the usual German thoroughness.

Cervallo found on experiment with guinea-pigs and frogs that narcotic doses of paraldehyde slightly slowed the breathing and heart-frequency. 'The relative bloodpressure was unchanged. Poisonous doses increased reflex irritability and occasioned death through respiratory paralysis. The cerebral hemispheres are principally affected, but also in part the medulla-oblongata and spinal marrow.

Doses of three to five grammes are followed in from five to fifteen minutes by a comfortable, sleepy feeling and soon by dreamless sleep which lasts for several hours. Reflex irritability is diminished, the pupils however react on being touched. The temperature falls a few tenths, and the pulse slows somewhat. The latter is shown by the sphygmograph to be moderately enlarged, with slightly increased tension. It is excreted entirely (unchanged) through the lungs.

Only after great doses, up to twelve grammes, are headache, vomiting, dizziness and other unpleasant results noticed. There is no rash. Usually waking is similar to that after natural sleep. There is no cumulative effect, but the system becomes accustomed

1 Continued from page 293.

2 All. Zeitschrift $f$. Psychiatrie 41stes Band 4tes and 5tes Heft. to its use. It can be continued and increased however without difficulty.

It is indicated in nervous and psychical conditions, in neurasthenia, hysteria, mania. It is less reliable in mental cases, with "angst-Vorstellungen." According to Gugl, in delirium tremens it has a sedative, as well as an hypnotic effect. A sedative effect is often seen in maniacal conditions. In the untidy, owing to the deep narcosis, the tendency to soil is increased.

In prescribing in the hospital, Stark uses R\%.- Paraldehyd 100.0, rum 150.0 , essent. citic 10.0, syr. simp. 300.0 , aq. 1400.0 . Of this 100.0 contains 5.0 of the paraldehyde. When not borne, or refused by the mouth it can be given as a clyster by the rectum with olive oil. Subcutaneously it has been given but little, as beside the burning and smarting of the place of injection, it has been followed by symptoms of collapse.

In the discussion of this paper Fürstner said he had found paraldelyyde most useful in agitated melancholia and in acute conditions. Jolly had got the best results in mania and delirium tremens.

\section{SUBCUTANEOUS EMPLOYMENT OF IRON IN MENTAL DISEASE. ${ }^{8}$}

W. Nasse states that various preparations of iron have been employed, such as the pyrophosphate with citrate of ammonia, dialyzed oxide of iron, phosphate of iron with citrate of soda, and albuminate of iron. Huguenin and Neuss used the first. The former in one case of pernicious anæmia, with good result. Neuss had severe local irritation with a third of his cases. Nasse also failed with this preparation. La Costa recommended the second preparation, but Neuss and Nasse found it entirely unfit for hypodermic use.

Martensen, Rosenthal and Neuss used the third, Rosenthal and Nasse found that it gave rise to local inflammation.

The fourth preparation has worked well in the hands of Doenitz and others. Nasse did not try it, as he found something better. This preparation, made by Finzelberg of Andernach, is called "Ferr. Oxid. Solub. pro injectione Finzelberg." It contains twentyfive per cent. of iron oxide.

A solution with water should be made; carefully brought to the boiling point and then immediately filtered. The idea was that the iron in a solution should not be precipitated by the alkaline blood. 'The dialyzed basic oxide of iron is dissolved in flesh peptone: dialyzed, then by evaporization converted into an easily soluble, red-brown powder, preserving its character for years.

Nasse used this preparation in twelve cases, for months at a time, usually injections being given twice daily. In five cases very slight irritation was caused, on isolated occasions and usually from neglect. In no case was any bad effect noticed, and in most, very decided benefit. The pulse and strength improved, and the mucous membrane became more red. Almost all the cases have recovered.

\section{HYOSCINE.}

Dr. Judson B. Andrews has recently written a communication on the use of this drug. ${ }^{4}$ There are two chemical combinations of this alkaloid, the hydrobromate and the hydriodate. The former is a white crys- 